\title{
The solution of Cauchy's Problem for linear partial differential equations, with constant coefficients, by means of integrals involving complex variables.
}

\author{
By C. A. Stewart.
}

(Received 5th February 1927. Read 4th March 1927.)

The object of this paper is to show how the theory of integrals involving complex variables may be applied to the integration of linear partial differential equations, possessing real, distinct characteristics and constant coefficients. The problem considered is a Cauchy problem (with analytic data)-typical of the equation of real characteristics and the method taken is that of Riemann. ${ }^{1}$ For simplicity of exposition, the second order hyperbolic equation is considered, but the results are given in such a form as to indicate an obvious generalisation to equations of higher order. ${ }^{2}$

The problem is to find that solution (known to be unique by Cauchy's existence theorem) of

1. $\left(\frac{\partial}{\partial x}+k_{1} \frac{\partial}{\partial y}\right)\left(\frac{\partial}{\partial x}+k_{2} \frac{\partial}{\partial y}\right) \boldsymbol{V}+A \frac{\partial \boldsymbol{V}}{\partial x}+B \frac{\partial \boldsymbol{V}}{\partial y}+C V=\phi(x, y)$

$\left\{\right.$ where $k_{1}, k_{2}, A, B, C$ are real constants, $\left.\left(k_{1} \neq k_{2}\right)\right\}$,

which is such that on the boundary specified by $x=\psi(y), V$ reduces to a given function $E_{0}(y)$, and $\frac{\partial V}{\partial x}$ to a given function $E_{1}(y)$.

It is sufficient for our present purpose to assume that $\phi, \psi, E_{0}, E_{1}$ are analytic in a region $\omega$, containing a portion $\sigma$ of the boundary; and it is necessary that $\sigma$ should not be tangential anywhere to a characteristic.

1 Darboux : Théorie générale des surfaces, II, pp. 75 et seq.

2 The Riemannian method of integration has been extended by the writer to equations of higher order: Proc. Lond. Math. Soc., 26 (1927). pp. 81-94. 
Let $P\left(x_{0}, y_{0}\right)$ be a point in $\omega$ near $\sigma$. Draw through $P$ lines of gradient $k_{1}, k_{2}$ to meet $\sigma$ in $R, Q$ respectively. (Fig. 1.)

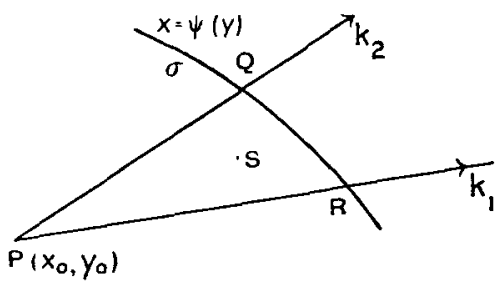

Fig. 1

$P$ can be taken sufficiently near the boundary to ensure that $Q R$ is a part of $\sigma$; and owing to the conditions we have imposed, no characteristic can meet $\sigma$ in more than one point. Any point $S$ in the area $P Q R$ may be taken as ${ }^{1}$

$$
x_{0}+u+v, y_{0}+k_{1} u+k_{2} v .
$$

The differential equation becomes

2. $\quad V_{u v}+a V_{u}+b V_{v}+c V=\phi\left(x_{0}+u+v, y_{0}+k_{1} u+k_{2} v\right)$

where $a=\frac{A k_{2}-B}{k_{2}-k_{1}}, b=\frac{A k_{1}-B}{k_{1}-k_{2}}, c=C$.

The boundary becomes $x_{0}+u+v=\psi\left(y_{0}+k_{1} u+k_{2} v\right)$, and there are similar changes in the forms of the boundary conditions.

Riemann's method consists substantially in integrating the expression

$$
\frac{\partial^{2}(\lambda V)}{\partial u \partial v}-\frac{\partial}{\partial u}\left\{\left(\lambda_{v}-a \lambda\right) V\right\}-\frac{\partial}{\partial v}\left\{\left(\lambda_{u}-b \lambda\right) V\right\}-\lambda \phi
$$

throughout the area $P, Q, R$ of the $u, v$ plane (Fig. 2), this expression being zero if $\lambda$ is a solution of the adjoint equation:

3.

$$
\lambda_{u v}-a \lambda_{u}-b \lambda_{v}+c \lambda=0
$$

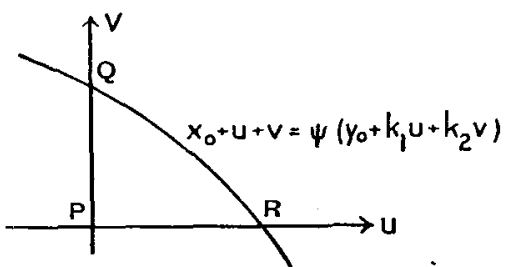

Fig. 2

1 If there is no term in $V_{x x}$ in the original equation, take the second order terms as $\frac{\partial}{\partial y}\left(\frac{\partial}{\partial x}+k_{1} \frac{\partial}{\partial y}\right) V$, and $x=x_{0},+u, y=y_{0}+k_{1} u+v$. 
It is shown that the integration provides the value of $V$ at $P$, viz., $V\left(x_{0}, y_{0}\right)$, if $\lambda$ can be found to satisfy the supplementary conditions :

$$
\lambda_{v}=a \lambda, \text { when } u=0 ; \lambda_{u}=b \lambda, \text { when } v=0 .
$$

This solution is usually given in terms of the Bessel Function of zero order, viz.,

4.

$$
\lambda=e^{a v+b u} J_{0}\{2 i \sqrt{ }\{u v(a b-c)\}\},
$$

but here we shall obtain $\lambda$ in the form of a double integral involving two complex variables. It will be seen later that this not only simplifies the subsequent integration but it also provides the obvious generalisation.

Consider the double integral

5.

$$
I=\frac{1}{(2 \pi \iota)^{2}} \iint_{\alpha \beta-\alpha \alpha-b \beta+c} \frac{e^{\alpha u+\beta v} d \alpha d \beta}{-\alpha,}
$$

where $\alpha, \beta$ are complex variables describing circles in their respective planes, given by $|\alpha|=R_{1}, \mid \beta=R_{2} . \quad R_{1}, R_{2}$ can clearly be taken so large that the function $\alpha \beta-a \alpha-b \beta+c$ does not vanish on the circles of integration (nor at points outside these circles). It is sufficient to take $R_{1}, R_{2}>R_{0}$, where $R_{0}$ is the positive root of the $R$-equation:

6.

For then

$$
R^{2}=\{|a|+|b|\} R+|c| \text {. }
$$

$$
\begin{aligned}
|\alpha \beta-a \alpha-b \beta+c| & \geqslant\left(R_{1}-R_{0}\right)\left(R_{0}-|a|\right)+\left(R_{2}-R_{0}\right)\left(R_{0}-|b|\right) \\
& +\left(R_{1}-R_{0}\right)\left(R_{2}-R_{0}\right)+\left\{R_{0}^{2}-(|a|+|b|) R_{0}-|c|\right\} . \\
\text { i.e. }>0 . & >0 .
\end{aligned}
$$

$I$ is an example of that class of double integrals, where the integrations with respect to $\alpha, \beta$ are independent of one another. The integrand is analytic and we can differentiate with regard to $u, v$ under the integral sign.

This gives

$$
\begin{aligned}
\frac{\partial^{2} I}{\partial u \partial v}-a \frac{\partial I}{\partial u}-b \frac{\partial I}{\partial v}+c I & =\frac{1}{(2 \pi \iota)^{2}} \iint e^{a u+\beta v} d \alpha d \beta \\
& =0
\end{aligned}
$$

since the integrand is everywhere analytic within the circles.

$$
\begin{aligned}
\operatorname{Again}^{*} I(u, 0) & =\frac{1}{(2 \pi \iota)^{2}} \iint \frac{e^{a u} d \alpha d \beta}{\alpha \beta-a a-b \beta+c} \\
& =\frac{1}{2 \pi \iota} \int \frac{e^{a u} d a}{a-b}=e^{b u} .
\end{aligned}
$$


Similarly $I(0, v)=e^{z v} ; I(0,0)=1$.

$I(u, v)$ is therefore the required solution $\lambda$; and we may easily deduce the other form of the result by taking $a=b+t_{1} ; \beta=a+t_{2}$.

Then $I(u, v)=\frac{e^{i u+a v}}{(2 \pi \imath)^{2}} \iint \frac{e^{u t_{1}+v t_{2}} d t_{1} d t_{2}}{t_{1} t_{2}-a b+c}$

over $\left|t_{1}+b\right|=R_{1} ;\left|t_{2}+a\right|=R_{2}$ and so enclosing the origins and points where $t_{1} t_{2}=a b-c$.

This gives $\frac{e^{b u+a v}}{2 \pi \iota} \int \frac{e^{u t_{1}+v / t_{1}(a b-c)} d t_{1}}{t_{1}}$

$$
=e^{i n+a v} J_{0}^{\dot{0}}(2 i \sqrt{ }\{u v(a b-c)\}) .
$$

The application of this result can now be best illustrated by taking the important case of a linear boundary; for even in the case of a curved boundary, the solution obtained will give an approximation in the neighbourhood. (i.e. when the tangent is taken as the first approximation to the bounding curve).

Take the boundary to be $x=h,{ }^{1}$ (except when $k_{1}$ or $k_{2}$ becomes infinite), and for definiteness assume $x_{0}<h$, (Fig. 3).
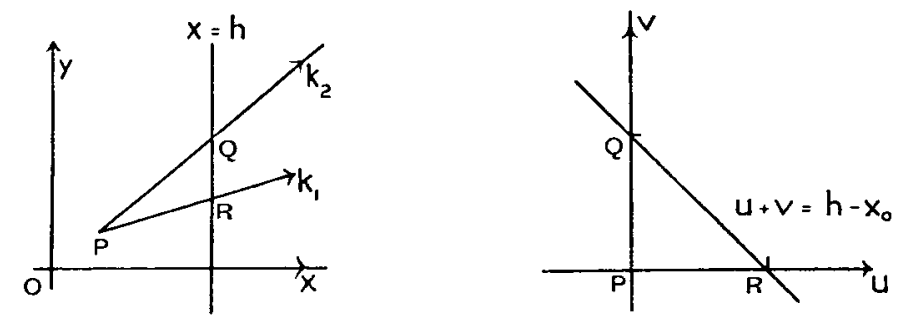

Fig. 3

The solution is provided by the integration of

7. $\iint\left[\frac{\partial^{2}(\lambda V)}{\partial u \partial v}-\frac{\partial}{\partial u}\left\{\left(\lambda_{v}-a \lambda\right) V\right\}-\frac{\partial}{\partial v}\left\{\left(\lambda_{u}-b \lambda\right) V\right\}-\lambda \phi\right] d u d v=\mathbf{0}$

where $A$ denotes the area $P Q R$.

The integration of the first term can be effected with regard to either $u$ or $v$ first, giving two forms of the result

8. $\begin{aligned} V\left(x_{0}, y_{0}\right) & =\int_{Q}^{R}\left[\lambda\left(V_{u}+b V\right) d u+\left(\lambda_{v}-a \lambda\right) V d v\right]-\lambda_{R} V_{R}+\iint_{A} \lambda \phi d u d v \\ & =\int_{Q}^{R}\left[-\lambda\left(V_{v}+a V\right) d v-\left(\lambda_{u}-b \lambda\right) V d u\right]-\lambda_{Q} V_{Q}+\iint_{A} \lambda \phi d u d v\end{aligned}$

1 Any linear boundary can be changed to $x=h$, by a suitable linear transformation of the independent variables. 
and so we may use them in a convenient combination, determined by the boundary conditions. Suppose we are given that on

$$
x=h, V=E_{0}(y), V_{x}=E_{1}(y) .
$$

These become:-

On $u+v=h-x_{0}, V=E_{0}\left(y_{0}+k_{1} u+k_{2} v\right), k_{2} V_{u}-k_{1} V_{v}=\left(k_{2}-k_{1}\right) E_{1}$.

Taking therefore $k_{2}$ times the first expression for the solution minus $k_{1}$ times the second, we find, (putting $d v=-d u$ )

9. $V\left(x_{0} y_{0}\right)=\iint_{A} \lambda \phi d u d v-\int_{Q}^{R} \lambda E_{1} d u+\int_{Q}^{R}\left(\frac{k_{1} \lambda_{u}-k_{2} \lambda_{v}-(a+b) \lambda}{k_{1}-k_{2}}\right) E_{0} d u$
$-\frac{k_{2}}{k_{1}-k_{2}} e^{b\left(h-x_{0}\right)} E_{0}\left\{y_{0}+k_{1}\left(h-x_{0}\right)\right\}-\frac{k_{1}}{k_{2}-k_{1}} e^{a\left(h-x_{0}\right)} E_{0}\left\{y_{0}+k_{2}\left(h-x_{0}\right)\right\}$.

The substitution of our value for $\lambda$ gives finally

10. $V\left(x_{0}, y_{0}\right)$

$$
\begin{aligned}
& =\frac{1}{(2 \pi \iota)^{2}} \iiint \int \frac{e^{a u+\beta c} \phi_{0}\left(x_{0}+u+v, y_{0}+k_{1} u+k_{2} v\right) d u d v d \alpha d \beta}{\alpha \beta-a \alpha-b \beta+c} \\
& -\frac{1}{(2 \pi \imath)^{2}} \iiint \frac{e^{a u+\beta v} E_{1}\left(y_{0}+k_{1} u+k_{2} v\right) d u d \alpha d \beta}{a \beta-a \alpha-b \beta+c} \\
& +\frac{1}{(2 \pi \iota)^{2}} \iiint \frac{e^{a u+\beta v}\left\{k_{1} a-k_{2} \beta-\left(k_{1}-k_{2}\right)(a+b)\right\} E_{0}\left(y_{0}+k_{1} u+k_{2} v\right) d u d \alpha d \beta}{\left(k_{1}-k_{2}\right)(\alpha \beta-a \alpha-b \beta+c)} \\
& -\frac{k_{2}}{k_{1}-k_{2}} e^{b\left(h-x_{0}\right)} E_{0}\left\{y_{0}+k_{1}\left(h-x_{0}\right)\right\}-\frac{k_{1}}{k_{2}-k_{1}} e^{\alpha\left(h-x_{1}\right)} E_{0}\left\{y_{0}+k_{2}\left(h-x_{0}\right)\right\} .
\end{aligned}
$$

The scope of the integrals has already been specified, and owing to the nature of the integrands, the integrations may be performed in any order. It will usually be simpler to integrate with respect to the real variables first, these being of the type

$$
\iint_{A} e^{a_{1} u+\beta_{1} v} F(u, v) d u d v, \int_{0}^{k} e^{\alpha_{1} u} G(u) \cdot d u
$$

and so, easily integrated if, for example, $F, G$ were exponential functions or polynomials. Take, therefore,

$$
\phi(x, y)=e^{\rho x+\sigma y}, E_{0}=e^{\theta_{1}, y}, E_{1}=e^{\theta}{ }^{\theta_{1} y} .
$$

Then from the solution obtained, we can deduce the corresponding solutions for polynomials.

We shall require certain results in integration which will be given a general form. 
11. (i)

$\iint-\int e^{\alpha_{1} u_{1}+\alpha_{:} u_{i}+\cdots+a_{n} u_{n}} d u_{1} d u_{2} \cdots d u_{n}=\frac{1}{2 \pi \iota} \int_{\gamma} \frac{e^{k t} d t}{t\left(t-\alpha_{1}\right)\left(t-\alpha_{2}\right) \cdots\left(t-\alpha_{\prime \prime}\right)}$

where on the left hand side, the integration extends to all positive values of $u_{1}, u_{2},--u_{n}$, satisfying the relation

$$
0 \leqslant u_{1}+u_{2}+\cdots+u_{n} \leqslant k \quad(k \text { positive })
$$

and on the right hand side, the contour $\gamma$ is the circle $|t|=R^{\prime}$, $\left(R^{\prime}>\max a_{r}\right)$.

12. (ii)

$\iint--\int e^{\alpha_{1} u_{1}+a_{2} u_{n}+\cdots+a_{n}{ }_{n}} d u_{1} d u_{2}-\cdot d u_{n-1}=\frac{1}{2 \pi \iota} \int_{\gamma} \frac{e^{k t} d t}{\left(t-a_{1}\right)\left(t-a_{2}\right)--\left(t-a_{n}\right)}$

where on the left hand side, the integration extends to all positive values of $u_{1}, u_{2},--u_{n-1}$, satisfying the relation

$$
0 \leqslant u_{1}+u_{2}+\cdots+u_{n-1} \leqslant k \text {, and } u_{n}=k-u_{1}-u_{2} \cdots-u_{n-1} .
$$

Denote the first integral on the left by $V(1,2, \cdots n)$ and the second by $S(1,2,-n)$.

(a) The substitution $u_{r}=k t_{r}(r=1, \cdots n)$ shows that $V$ and its first $(n-1)$ differential coefficients with regard to $k$, vanish for $k=0$.

(b) The change of variables from

$$
\begin{gathered}
u_{1}, u_{2}, \cdots, u_{\imath} \text { to } \\
u_{1}, u_{2}, \cdots-u_{n-1}, \theta\left(=u_{1}+u_{2}+\cdots+u_{n}\right)
\end{gathered}
$$

gives

$$
V(1,2, \cdots n)=\int_{0}^{k} F(\theta) d \theta
$$

where

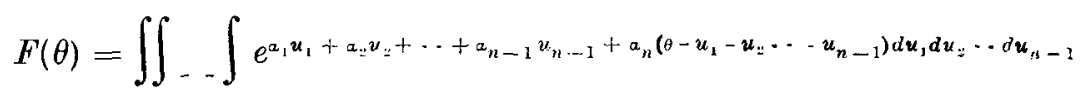

taken over all positive values of $u_{1}, u_{2},--u_{n-1}$ satisfying

$$
\begin{aligned}
0 \leqslant u_{1}+u_{2}+\cdots+u_{n-1} \leqslant \theta \quad(0 \leqslant \theta \leqslant k) \\
\therefore \quad \frac{d V(1,2,-n)}{d k}=F(k)=S(1,2,-n) .
\end{aligned}
$$


(c) Integration of $V(1,2,-n)$, in the original form, with respect to $u_{n}$ gives

$$
\begin{aligned}
& V(1,2,-n)=\frac{S(1,2,-n)}{\alpha_{n}}-\frac{V(1,2,-n-1)}{\alpha_{n}} \\
\therefore \quad & \left(\frac{d}{d k}-\alpha_{n}\right) V(1,2,-n)=V(1,2,-n-1) .
\end{aligned}
$$

We note that $V_{1}=\frac{e^{a_{1} k}}{a_{1}}-\frac{1}{a_{1}}$ and we therefore interpret $S_{1}$ to mean $e^{a, k}, V_{0}=1, S_{0}=0$, and deduce by continued application of the above result that

13.

$$
\left(\frac{d}{d k}-a_{1}\right)\left(\frac{d}{d k}-a_{2}\right) \cdots\left(\frac{d}{d k}-a_{n}\right) V(1,2,-n)=1 .
$$

$V(1,2,-n)$ is therefore that solution of equation 13 which satisfies the initial conditions.

$$
V=0, \frac{d V}{d k}=0,-\frac{d^{n-1} V}{d k^{n-j}}=0, \text { when } k=0 .
$$

The contour integral

$$
\frac{1}{2 \pi \imath} \int_{\gamma} \frac{e^{k t} d t}{t\left(t-a_{1}\right)-\cdots\left(t-\alpha_{n}\right)}
$$

gives this solution, for the result of substituting this on the left hand side of equation 13. Gives

$$
\frac{1}{2 \pi \iota} \int_{\gamma} e^{k t} \frac{d t}{t}=1
$$

Also putting $k=0$ in the contour integral above and in those obtained by differentiating once, twice up to $(n-1)$ times will give integrands of the type

$$
\frac{t^{m}}{t\left(t-\alpha_{1}\right)\left(t-a_{2}\right) \cdots\left(t-\alpha_{n}\right)} \quad(m=0,1,-n-1)
$$

These do not possess singularities at infinity and therefore vanish.

By differentiation we get

$$
S(1,2,-n)=\frac{1}{2 \pi \iota} \int_{\gamma} \frac{e^{k t} d t}{\left(t-a_{1}\right)\left(t-a_{2}\right) \cdots\left(t-\alpha_{n}\right)} .
$$

In the solution for $V\left(x_{0}, y_{0}\right)$, the part depending on $\phi$ is

$$
\frac{1}{(2 \pi \iota)^{2}} \iiint \int \frac{e^{a u+\beta v+p\left(x_{0}+u+v\right)+\sigma\left(y_{0}+k_{1} u+k_{z} v\right)} d u d v d \alpha d \beta}{\alpha \beta-a \alpha-b \beta+c}
$$

over

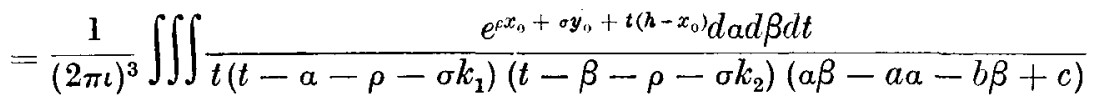

$$
\begin{aligned}
& \text { for }|t|=R^{\prime},|\alpha|=R_{1},|\beta|=R_{2} \text {. }
\end{aligned}
$$


Now take $R^{\prime}$ so large that $\left|t-\rho-\sigma k_{1}\right|>R_{1}, R_{2}$

$$
\text { and }\left|t-\rho-\sigma k_{2}\right|>R_{1}, R_{2} \text {. }
$$

Then, in integrating with respect to $\alpha, \beta$, we note that the integrand possesses a singularity only at one place outside the circles

$$
|a|=R_{1},|\beta|=R_{2} \text {, viz., where } a=t-\rho-\sigma k_{1}, \beta=t-\rho-\sigma k_{2}
$$

and so the above integral becomes

$$
\begin{gathered}
\left.\frac{1}{2 \pi \iota} \int \frac{e^{\rho x_{0}+\sigma y_{0}+t\left(h-x_{0}\right)} d t}{t\left\{\left(t-\rho-\sigma k_{1}\right)\left(t-\rho-\sigma k_{2}\right)\right.}-a\left(t-\rho-\sigma k_{1}\right)-b\left(t-\rho-\sigma k_{2}\right)+c\right\} \\
=\frac{1}{2 \pi \iota} \int \frac{e^{\rho r_{0}+\sigma y_{0}+t\left(h-x_{0}\right)} d t}{t f(\rho-\overline{t, \sigma)}}
\end{gathered}
$$

where $f\left(D_{1}, D_{2}\right) \Gamma^{\top}=\phi$ is the original differential equation $1, D_{1}, D_{2}$ denoting the operators $\frac{\partial}{\partial x}, \frac{\partial}{\partial y}$. to be

The part of the solution depending on $E_{1}$ is proved similarly

$$
-\frac{1}{2 \pi \iota} \int \frac{e^{\theta_{1} \nu_{\mathrm{o}}+t\left(h-x_{0}\right)} d t}{f\left(-t, \theta_{1}\right)}
$$

The part of the solution depending on $E_{0}$ is

$$
\begin{gathered}
\frac{1}{(2 \pi \iota)^{2}} \iiint \frac{e^{\alpha u+\beta v}\left\{k_{1} a-k_{2} \beta-\left(k_{1}-k_{2}\right)(a+b)\right\} e^{\theta_{0}\left(y_{0}+k_{1} u+k_{2} v\right)} d u d \alpha d \beta}{\left(k_{1}-k_{2}\right)(\alpha \beta-a \alpha-b \beta+c)} \\
-\frac{k_{2}}{k_{1}-k_{2}} e^{b\left(h-x_{0}\right)+\theta_{0} y_{0}+\theta_{0}\left(h-x_{0}\right) k_{1}}-\frac{k_{1}}{k_{2}-k_{1}} e^{n\left(h-x_{0}\right)+\theta_{0} y_{0}+\theta_{0}\left(h-x_{0}\right) k_{z}}
\end{gathered}
$$

The part of this involving the integral is

$\frac{1}{(2 \pi \iota)^{3}} \iiint \frac{\left\{k_{1} \alpha-k_{2} \beta-\left(k_{1}-k_{2}\right)(a+b)\right\} e^{\theta_{0} y_{0}+t\left(h-x_{0}\right)} d t d \alpha d \beta}{\left(k_{1}-k_{2}\right)\left(t-\alpha-\theta_{0} k_{1}\right)\left(t-\beta-\theta_{0}\right.} \overline{\left.k_{2}\right)(\alpha \beta-a \alpha-b \beta+c)}$

But in this integral, there are 3 places outside $|\alpha|=R_{1},|\beta|=R_{2}$ where the integrand has a singularity

(i) $a=t-\theta_{0} k_{1}, \beta=t-\theta_{0} k_{2} \quad$ (ii) $\alpha=\infty, \beta=t-\theta_{0} k_{2} \quad$ (iii) $a=t-\theta_{0} k_{1}, \beta=\infty$ $(\alpha=\infty, \beta=\infty$ is not a singularity).

The first gives $\frac{1}{2 \pi \iota} \int \frac{\left\{t-\left(k_{1}+k_{2}\right) \theta_{0}-a-b\right\}}{f\left(-t, \theta_{0}\right)} e^{\theta_{0} y_{0}+t\left(h-x_{1}\right)} d t$.

The second gives $-\frac{k_{1}}{k_{1}-k_{2}} e^{\theta_{0} y_{0}+\left(a+\theta_{0} k_{0}\right)\left(h-x_{0}\right)}$.

The third gives $\frac{k_{2}}{k_{1}-k_{2}} e^{\theta_{0} y_{0}+\left(b+\theta_{0} k_{1}\right)\left(h-x_{0}\right)}$. 
The part, therefore, depending on $E_{0}$ is simply

$$
\frac{1}{2 \pi \iota} \int \frac{\left\{t-\left(k_{1}+k_{2}\right) \theta_{0}-a-b\right\} e^{\theta_{0} y_{0}+t\left(h_{l}-x_{0}\right)} d t}{f\left(-t_{1} \theta_{0}\right)} .
$$

The solution ${ }^{1}$ is therefore given by

14. $V\left(x_{0} y_{0}\right)$

$$
=\frac{1}{2 \pi \imath} \int\left[\frac{e^{\rho x_{0}+\sigma y_{0}}}{t f(\rho-t, \sigma)}-\frac{e^{\theta_{1} y_{0}}}{f\left(-t, \theta_{1}\right)}+\frac{\left\{t-\left(k_{1}+k_{2}\right) \theta_{0}-A\right\}}{f\left(-t, \theta_{0}\right)} e^{\theta_{1, \prime_{0}}}\right] e^{t\left(h-x_{1,}\right)} d t .
$$

This solution obviously applies to equations of elliptic or parabolic type, although the method of obtaining it is peculiar to the hyperbolic type.

\section{Examples:}

(i) Hyperbolic type:

Find the solution of

$$
V_{x x}-3 V_{x y}+2 V_{y y}+2 V_{x}+4 V_{y}+V=1
$$

which is such that $V_{x}=y, V=0$ when $x=0$.

The first part is

$$
\begin{aligned}
\frac{1}{2 \pi \iota} \int \frac{e^{-t x_{0}} d t}{t(t-1)^{2}} & =1+\text { coefficient of } \frac{1}{T} \text { in } \frac{e^{-x_{1,1}(1+T)}}{T^{2}} \\
& =1-x_{0} e^{-x_{0}}
\end{aligned}
$$

The second part is obtained from

$$
\begin{aligned}
& -\frac{1}{(2 \pi \iota)} \int \frac{e^{\theta_{1}^{-} y+t\left(h-x_{0}\right)} d t}{t^{2}+t\left(3 \theta_{1}-2\right)+\left(2 \theta_{1}^{2}+4 \theta_{1}+1\right)} \text { which gives } \\
& \frac{2 e^{\theta_{1} y_{0}-x_{0}\left(1-3 \theta_{1}\right)} \sinh \frac{x_{0}}{2} \sqrt{ }\left(\theta_{1}^{2}-28 \theta_{1}\right)}{\sqrt{ }\left(\theta_{1}^{2}-28 \theta_{1}\right)} .
\end{aligned}
$$

The coefficient of $\theta_{1}$ in this is $e^{-x_{1}}\left(x_{0} y_{0}+\frac{3}{2} x_{0}{ }^{2}-\frac{7}{6} x_{0}{ }^{3}\right)$.

The third part is zero.

The required solution is $1+e^{-x}\left(x y+\frac{3}{2} x^{2}-\frac{7}{3} x^{3}-x\right)$.

1 Cf. Zeilon: Arkev für Matematik, Astronomi och Fysik, 6 (1910). 
(ii) Parabolic type:

Find the solution of $\frac{\partial^{2} V}{\partial x^{2}}=\frac{\partial V}{\partial y}$, satisfying

$$
V=A, V_{x}=B y \text { on } x=0
$$

1st part is zero:

2nd part is the coefficient of $\theta_{1}$ in the expansion of

$$
-\frac{B}{2 \pi \iota} \int \frac{e^{\theta_{1} y_{0}-t x_{i}} d t}{t^{2}-\theta_{1}}
$$

that is in $\frac{B e^{\theta_{1} y_{1 "}} \sinh x_{0} \sqrt{ } \theta_{1}}{\sqrt{ } \theta_{1}}$ and therefore is $B x_{0}\left(y_{0}+x_{0}{ }^{2} / 6\right)$.

3rd part is $\frac{A}{2 \pi \iota} \int \frac{t e^{-t x_{0}} d t}{t^{2}}=A$.

Solution is $A+B x\left(y+x^{2} / 6\right)$.

(iii) Elliptic type:

Find the solution of $\frac{\partial^{2} V}{\partial x^{2}}+\frac{\partial^{2} V}{\partial y^{2}}=c V$ satisfying $V=A \sin p y$, $V_{x}=0$, when $x=0$.

Corresponding to $E_{0}=e^{\iota p y}, E_{1}=0$, the solution is

$$
\begin{gathered}
\frac{1}{2 \pi \iota} \int \frac{t e^{p y_{*}-t x_{*}} d t}{t^{2}-p^{2}-c} \\
=A e^{\iota p y_{0}} \cosh x_{0} \sqrt{ }\left(p^{2}+c\right) .
\end{gathered}
$$

Therefore the solution is

$$
A \sin p y \cosh x \sqrt{ }\left(p^{2}+c\right) .
$$

